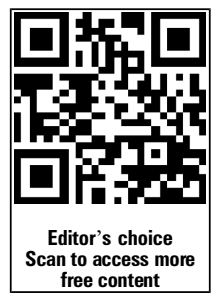

Department of Health Sciences, University of Leicester, Leicester, UK

\section{Correspondence to} Professor Graham P Martin, Department of Health Sciences, University of Leicester, 22-28 Princess Road West, Leicester LE1 6TP, UK: gpm7@le.ac.uk

Received 2 July 2014 Accepted 3 July 2014 Published Online First 18 July 2014

\section{SLinked}

- http://dx.doi.org/10.1136/ bmjas-2013-002483

- http://dx.doi.org/10.1136/ bmjqs-2013-002688

- http://dx.doi.org/10.1136/ bmjqs-2013-002685

- http://dx.doi.org/10.1136/

bmjqs-2013-002534

- http://dx.doi.org/10.1136/

bmjqs-2013-002643

- http://dx.doi.org/10.1136/

bmjqs-2013-002581

- http://dx.doi.org/10.1136/

bmjqs-2013-002718

CrossMark

To cite: Martin GP, DixonWoods M. BMJ Qual Saf 2014:23:706-708.

\title{
After Mid Staffordshire: from acknowledgement, through learning, to improvement
}

\author{
Graham P Martin, Mary Dixon-Woods
}

For many readers, the story of the quality of care at Mid Staffordshire NHS Trust in the UK from 2005 to 2009 will need little introduction. The substandard care provided and the combination of circumstances that allowed such a situation to persist for several years have received widespread attention in the general media as well as in healthcare journals. ${ }^{1-4}$ At the heart of this coverage are the findings of two inquiries led by Sir Robert Francis QC-the first focusing on the quality of care provided at Stafford Hospital $^{5}$ and the second on the role of a wider system of governance that failed to identify and remedy the problems over a sustained period. ${ }^{6}$

The inquiries have had a far-reaching impact within the UK. Some of the responses have been measured and considered; some less so. Media coverage has focused on the question of how such egregious failings could have gone unchecked for so long and on the extent to which they may be present in other NHS hospitals. The political impact has also been profound, with Mid Staffordshire characterised by the secretary of state for health as an extreme example of the "crisis in standards of care' that pervades the health and social care system. ${ }^{7}$ In response to the second inquiry, the British government instigated two further reviews, one focusing on the quality of care at other hospitals with unexpectedly high adjusted mortality rates, ${ }^{8}$ and the other seeking to begin the task of translating the 290 recommendations offered by Francis into concrete plans for the Service. ${ }^{9}$

The texts of past inquiries show that Mid Staffordshire is not a unique case historically ${ }^{10}$; neither are healthcare failings confined to any particular health system in the present. While the scale and scope of poor-quality care at Stafford may have been exceptional, 'dark spots' and 'bright spots' in the quality of care can be found across the NHS ${ }^{11}$ and probably in every healthcare system. It is tempting to attribute such dark spots to neglectful or malevolent individuals and their acts of commission or omission. However, as Francis's second inquiry shows, such analyses offer only a partial and distorted understanding of a scandal that was the product of the intersection of institutional, structural, behavioural and cultural factors. The first step towards learning the lessons of Stafford, and preventing history from repeating itself once more, is the rejection of straightforward solutions that present the hospital as a one-off outlier or locate the problem in 'bad-apple' cliniciansbut also to reject analyses that suggest that such occurrences are inevitable and unavoidable.

With this objective in mind, BMJ Quality \& Safety commissioned a number of perspective papers. This series of seven peer-reviewed commentaries comes from diverse standpoints, including not just the usual academic and clinical fields typically represented in the journal, but also others such as sociolegal studies. Each contribution reflects on the scandal (the events at Mid Staffordshire themselves), the ensuing inquiries and what can be learnt from them. The emphasis in each is highly practical: while they focus on different levels of the system, they are united in their eagerness to use the example of Mid Staffordshire to help healthcare to improve.

The series begins with a contribution from Sidney Dekker and Thomas Hugh. ${ }^{12}$ Examining some of the more heated media reactions to Stafford, they highlight the inadequacy of accounts that search for a single cause of incidences of 
suffering in healthcare. Such accounts can never do justice to the multifactorial reality of harm in complex systems, and while identifying an ultimate focus for blame may be analytically (perhaps even emotionally) satisfying, it offers a weak basis for understanding, reconciliation and improvement. If any effort to prevent recurrence is to succeed, argue Dekker and Hugh, it must be premised on an acknowledgement that risk is an inevitable by-product of healthcare complexity: efforts to mitigate that risk must focus on the system, and frameworks of accountability must recognise the ethical dilemmas and trade-offs with which clinical staff must contend every day.

Two contributions focus on the legal framework governing healthcare provision, addressing the legislative recommendations put forward by the second inquiry. In their piece, Karen Yeung and Jeremy Horder consider Francis's recommendation that a new criminal offence be introduced for occasions where wilful mistreatment has given rise to a breach of fundamental standards of care. ${ }^{13}$ This proposal has been criticised by some and might be seen as exemplifying the individualisation of culpability challenged by Dekker and Hugh. ${ }^{12}$ But Yeung and Horder suggest that such a measure would address an important anomaly in the current English criminal law relating to the criminal sanctions available for wilful neglect of those lacking mental capacity compared with those who do not lack capacity. This gap, they argue, undermines the expressive function of criminal law: the offence of wilful neglect is likely to be invoked only very rarely, but for Yeung and Horder it plays a critical role in declaring the values and standards that are foundational to professional and organisational conduct and should not give rise to a culture of fear, as critics have suggested.

Meanwhile, Oliver Quick's article focuses on another contested legislative recommendation from the second Francis inquiry, namely the creation of a statutory duty of candour on healthcare organisations and professionals. ${ }^{14}$ Once again, the potential unintended consequences of such a heavy instrument of change have been highlighted in popular and academic exchanges on this subject, but like Yeung and Horder, Quick highlights the normative contribution that legislative change can make to the culture of healthcare provision. Arguing that the duty should apply to individual professionals as well as organisations, he suggests that 'the honesty and care displayed by open disclosure has the potential for strengthening and not weakening trust relationships': a duty of candour, as part of a wider set of measures designed to investigate and redress incidences of harm, might contribute to the more mature approach to accepting risk, thus facilitating the reconciliation and learning envisaged by Dekker and Hugh. ${ }^{12}$

The prolonged nature of the situation at Mid Staffordshire and the apparent inaction by clinicians, management and regulators have been the subject of much soul-searching following the second inquiry and are addressed directly by two of the contributions. Aled Jones and Daniel Kelly challenge the widely held view that clinical staff at Stafford were silent in the face of the harm they witnessed, ${ }^{15}$ pointing out that in fact, multiple efforts were made to raise concernsbut these were unheard, ignored or actively suppressed. Jones and Kelly argue that the common distinction made between 'whistleblowing' and 'organisational silence' is an unhelpful binary, and that a key task for healthcare organisations is to find ways to solicit and listen to the myriad means by which staff might raise concerns. Organisations that fail in this task risk generating a negative feedback loop, in which clinicians' experiences of being ignored give rise to a nihilistic culture where no one can see the point of speaking up. Instruments such as cultural barometers, they suggest, might help managers to evaluate the relationships between 'sharp-end' and 'blunt-end' staff, and take remedial action if the culture is a negative one.

In a similar vein, Carl Macrae looks at the long 'incubation period' that preceded the revelations at Stafford and other disasters inside and outside healthcare. ${ }^{16} \mathrm{He}$ asks whether more might be done to gather and make sense of the warning signs that, while weak and ambiguous, nevertheless point towards a gathering storm. For healthcare organisations, this means finding ways of identifying and filling gaps in knowledge about quality and risk - 'problem-sensing' rather than 'comfort-seeking' behaviour ${ }^{11}$-and a smarter approach to monitoring quality that pays particular heed to anomalous and disconfirming data. For the wider healthcare system, Macrae recommends independent safety investigation organisations, similar to the UK Air Accidents Investigation Branch or Rail Accident Investigation Branch, whose role is clearly distinct from regulation, commissioning or performance management and relates solely to understanding causes of failures and disseminating recommendations. Following the abolition of National Patient Safety Agency, the UK has no such body, such that investigations are localised, partial and reactive, punctuated only sporadically by judicial inquiries when popular outcry occasions them. In consequence, 'healthcare systems lack a routine and independent source of knowledge on the processes that lead to systemic failures of care, the kinds of warnings signs that managers and regulators should remain vigilantly attentive to and afraid of, and the location of potential pockets of ignorance in healthcare organisations and the system as a whole.'

Some of the responses to Francis will undoubtedly require primary or secondary legislation, or high-level policy intervention beyond the control of clinicians and healthcare managers. Even Jones and Kelly ${ }^{15}$ and Macrae's ${ }^{16}$ calls for greater vigilance to avert disaster 
may sound dauntingly labour-intensive and challenging to operationalise to those grappling with the day-to-day realities of healthcare delivery in an increasingly complex and financially challenged system. Our series therefore concludes with two contributions that are overtly practical in orientation, offering a number of (comparatively) easy wins that lay down the gauntlet to practitioners, demanding that they take ownership of quality and safety and, as one puts it, make 2014 'the year that improving safety and quality went 'mainstream'.' Drawing on their experience as National Medical Director's Fellows, Parashar Ramanuj and colleagues offer a rallying cry to junior clinicians of every profession, and a framework whereby organisations might act on the insights into quality of care offered by their junior staff. ${ }^{17}$ Following the process used by the post-Francis Keogh review of 14 organisations with persistently high mortality rates, ${ }^{8}$ they argue for the value of 'peer-led inspections' that use a combination of methods to access and synthesise the insights of a full range of sharp-end staff. Such an approach offers one possible means of accessing the 'soft intelligence' that is, as Jones and Kelly show, so easily forsaken. Ramanuj et al also advocate its value in nurturing a culture of collaboration, empowerment and self-efficacythough they acknowledge that it rests on the existence of a body of senior managers and clinicians who are prepared to take the input of their junior colleagues seriously.

Finally, using examples in the academic and practice literature, Patricia Woodhead and colleagues outline four levels of action-from the individual patient and clinician upwards-and four principles of intervention that can help to turn good intentions into better outcomes and move from isolated projects and patchy quality of care towards sustained, widely spread improvement. ${ }^{18}$ Highlighting the importance of measurement and feedback, focused and professional leadership, commissioning frameworks that value quality, and action to ensure a nurturing culture, they make clear suggestions for everyone involved at every level of the system to start to overcome the inertia that can follow a crisis of Stafford's scale.

The contributions in this series offer thoughtful reflections and practical suggestions. They will help in responding to the call made by Donald Berwick and colleagues on the National Advisory Group on the Safety of Patients in England for the NHS to respond to Mid Staffordshire by striving to 'become, more than ever before, a system devoted to continual learning and improvement of patient care, top to bottom and end to end. ${ }^{9}$ The challenge is enormous, and it confronts health systems everywhere. If the crisis of Mid Staffordshire is not to be wasted, the opportunity must be seized now by everyone with a responsibility for quality and safety of care.
Contributors Both authors conceived the idea for the series and the article together. GPM led the writing of the article, and MDW made critical contributions to it. Both authors approved the final version of the article.

Competing interests GPM and MDW are editors at BMJ Quality \& Safety. MDW acknowledges her role as a member of the National Advisory Group on the Safety of Patients in England, and a Wellcome Trust Senior Investigator Award (reference WT097899MA) which supported her contribution to this editorial.

Provenance and peer review Not commissioned; internally peer reviewed.

\section{REFERENCES}

1 Newdick C, Danbury C. Culture, compassion and clinical neglect: probity in the NHS after Mid Staffordshire. J Med Ethics 2013; in press

2 Ross F. Having the courage to care: lessons learnt after Mid Staffordshire. Br J Nurs 2013;22:296.

3 McLellan A. Mid Staffordshire inquiry: too long, too late, but Francis can still help make the NHS better. Health Serv J 2013;123:3

4 Templeton S-K. Labour failure 'let hundreds die' in hospital. The Sunday Times 27 January 2013.

5 Mid Staffordshire NHS Foundation Trust Inquiry. Independent Inquiry into care provided by Mid Staffordshire NHS Foundation Trust. London: The Stationery Office, 2010.

6 Mid Staffordshire NHS Foundation Trust Public Inquiry. Report of the Mid Staffordshire NHS Foundation Trust Public Inquiry: executive summary. London: The Stationery Office, 2013.

7 Department of Health. Hunt orders review into OFSTED style ratings for NHS and social care (press release) [Internet]. 2012 [cited 2014 Apr 8]. https://www.gov.uk/government/news/huntorders-review-into-ofsted-style-ratings-for-nhs-and-social-care

8 Keogh B. Review into the quality of care and treatment provided by 14 hospital trusts in England: overview report. London: NHS, 2013.

9 National Advisory Group on the Safety of Patients in England. A promise to learn-a commitment to act: improving the safety of patients in England. London: Department of Health, 2013.

10 Walshe K. Inquiries: learning from failure in the NHS? London: Nuffield Trust, 2003.

11 Dixon-Woods M, Baker R, Charles K, et al. Culture and behaviour in the English National Health Service: overview of lessons from a large multimethod study. BMJ Qual Saf 2014;23:106-15.

12 Dekker SWA, Hugh TB. A just culture after Mid Staffordshire. BMJ Qual Saf 2014;23:356-8.

13 Yeung K, Horder J. How can the criminal law support the provision of quality in healthcare? BMJ Qual Saf 2014;23:519-24.

14 Quick O. Regulating and legislating patient safety: the case for candour. BMJ Qual Saf 2014;23:614-18.

15 Jones A, Kelly D. Deafening silence? Time to reconsider whether organizations are silent or deaf when things go wrong. BMJ Qual Saf 2014;23:709-13.

16 Macrae C. Early warnings, weak signals and learning from healthcare disasters. BMJ Qual Saf 2014;23:440-5.

17 Ramanuj PP, Ryland H, Mitchell EW, et al. In the spotlight: healthcare inspections as an opportunity for trainee clinicians to be the leaders of today. BMJ Qual Saf 2014;23:624-8.

18 Woodhead T, Lachman P, Mountford J, et al. From harm to hope and purposeful action: what could we do after Francis? BMJ Qual Saf 2014;23:619-23. 